\title{
OS RISCOS DA VIDA SEXUAL ATIVA NA ADOLESCÊNCIA: O RELATO DE UMA CAPACITAÇÃO EM UMA ESTRATÉGIA DE SAÚDE DA FAMÍLIA
}

\author{
Gabriella Ramos Chagas \\ Faculdades Integradas Pitágoras de Montes Claros \\ gabiramos_97@hotmail.com \\ Isabella Medeiros de Lima \\ Faculdades Integradas Pitágoras de Montes Claros \\ bellaa1303@hotmail.com \\ Lucas Roberto de Souza Botelho \\ Faculdades Integradas Pitágoras de Montes Claros \\ lucas.roberto@me.com
}

\author{
Nathália Pádua Pereira \\ Faculdades Integradas Pitágoras de Montes Claros \\ nathaliaa_p@hotmail.com \\ Betânia Maria Araujo Passos \\ Universidade Estadual de Montes Claros \\ betahidro26@yahoo.com.br \\ Brunna Librelon Costa \\ Universidade Estadual de Montes Claros \\ bu.librelon@yahoo.com.br
}

\section{Resumo}

A saúde sexual e o planejamento familiar são temas importantes a serem tratados entre adolescentes. Este trabalho objetivou descrever a experiência referente à uma capacitação acerca dos riscos da vida sexual ativa na adolescência numa Estratégia de Saúde da Família (ESF) em Montes Claros, MG. Trata-se de um estudo descritivo do tipo relato de experiência sobre ações desenvolvidas por acadêmicos de medicina durante sua intervenção direcionada a Agentes Comunitários de Saúde (ACS) no mês de dezembro de 2016. Foram desenvolvidas atividades de educação sexual, distribuídos panfletos informativos com ênfase em Doenças Sexualmente Transmissíveis (DST) e gravidez indesejada. Averiguou-se que os agentes não possuíam conhecimento suficiente acerca do tema e devem intervir mais ao se depararem com jovens em situação de risco de adquirir DST ou gravidez não planejada. Salienta-se a importância dos ACS no que se refere à promoção da educação em saúde e gravidez.

Palavras-chave: Doenças Sexualmente Transmissíveis. Gravidez. Adolescência. Estratégia de Saúde da Família. Educação em Saúde.

\section{THE RISKS OF ACTIVE SEXUAL LIFE IN ADOLESCENCE: THE REPORT OF A QUALIFICATION IN A FAMILY HEALTH STRATEGY}

Abstract

Sexual health and family planning are important issues to be treated between adolescents. This work aimed to describe the experience regarding a training about the risks of active sexual life in adolescence in a Family Health Strategy in Montes Claros, MG. This is a descriptive study, an experience report, about actions developed by academics from the medical course during their intervention directed to Community Health Agents (CHA) in december, 2016. Sex education activities were developed and informational pamphlets with emphasis on Sexually Transmitted Diseases (STD) and unwanted pregnancies were distributed. It was found that the agents did not have enough knowledge on the subject and should intervene more when confronted with young people at risk of acquiring STD or unplanned pregnancy. The importance of CHWs in promoting health and pregnancy education is highlighted.

Keywords: Sexually Transmitted Diseases. Pregnancy. Adolescence. Family Health Strategy. Health education.

\section{LOS RIESGOS DE LA ACTIVIDAD SEXUAL EN LA ADOLESCENCIA: EL INFORME DE UNA FORMACIÓN EN UNA ESTRATEGIA DE SALUD FAMILIAR} Resumen

La salud sexual y la planificación familiar son cuestiones importantes que deben abordarse en los adolescentes. Este estudio tuvo como objetivo describir la experiencia relativa a una formación sobre los riesgos de la actividad sexual en la adolescencia en una Estrategia de Salud Familiar en Montes Claros, Minas Gerais. Se trata de un estudio descriptivo del tipo relato de experiencia sobre acciones desarrolladas por académicos de medicina durante su intervención dirigida a los Agentes Comunitarios de Salud (ACS) en diciembre de 2016. Se desarrollaron actividades de educación sexual, distribuidos folletos informativos con énfasis en Enfermedades Sexualmente Transmisibles (EST) y embarazos no deseados. Se verificó que los agentes no poseían conocimiento suficiente sobre el tema y deben intervenir más al encontrarse con jóvenes en situación de riesgo de adquirir EST o embarazo no planificado. Se destaca la importancia de los ACS en lo que se refiere a la promoción de la educación en salud y el embarazo.

Palavras clave: Enfermedades Sexualmente Transmisibles. Embarazo. Adolescencia. Estrategia de Salud de la Familia. Educación en Salud. 
Os riscos da vida sexual ativa na adolescência: o relato de uma capacitação em uma Estratégia de Saúde da Família

\section{INTRODUÇÃO}

A adolescência, transição da infância para a vida adulta, é um período marcante no desenvolvimento humano, pois compreende fortes transformações biológicas, emocionais, cognitivas e sociais. Nesta fase, o adolescente inicia sua independência e autonomia, apresentando novos comportamentos e vivenciando novas experiências (GONÇALVES; KNAUTH, 2006; IBGE, 2016). A iniciação precoce na vida sexual é uma conduta importante a ser estudada, tendo em vista que adolescentes sem o devido conhecimento dos riscos da atividade sexual desprotegida podem estar suscetíveis a Doenças Sexualmente Transmissíveis (DST) e a gravidez não planejada (SAWYER et al., 2012; SÀNCHEZ, BERTOLOZZI, 2007; CRUZEIRO et al., 2010).

O aumento da atividade sexual entre adolescentes levou a um aumento no índice de gravidez não planejada (GRADIM, FERREIRA, MORAES, 2010), e estima-se que mais de 10 milhões de infectados pelo vírus da AIDS estejam na faixa etária entre 15 e 24 anos, mostrandose um problema de saúde pública (MINISTÉRIO DA SAÚDE, 2013). Dados da Pesquisa Nacional de Saúde Escolar de 2015 (IBGE, 2016) indicam que um percentual de 19,3\% meninas e 34,5\% de meninos de 13 a 15 anos já tiveram relação sexual, o que se torna preocupante tendo em vista que as ações assumidas nessa etapa da vida tendem a ser mantidas na fase adulta, podendo trazer consequências como o acometimento de doenças crônicas, prejudicando a qualidade de vida. Outro estudo ressalta que a vulnerabilidade dos adolescentes é composta, dentre outros fatores, pela iniciação precoce na vida sexual, por dúvidas sobre a transmissão do HIV e a não utilização efetiva do preservativo (CHAVES, 2014).

Frente a vários desafios, a Estratégia de Saúde da Família (ESF) exerce papel importante na promoção e educação em saúde da população local. No Brasil, a ESF é composta por uma equipe multiprofissional que possui, ao menos, Médico, Enfermeiro, Auxiliar ou Técnico de Enfermagem e Agentes Comunitários de Saúde (ACS), tendo por objetivo garantir a todos o acesso à uma atenção básica de saúde de qualidade. A ESF é responsável por traçar o perfil epidemiológico das comunidades e, a partir dele, propor formas de intervenção à saúde local por meio, principalmente, da prevenção, respeitando os princípios do Sistema Único de Saúde (SUS). Nesse sentido, o principal responsável da equipe por conhecer o perfil das comunidades e de cada família pertencente a elas é o Agente Comunitário de Saúde (ACS), que é um profissional que se identifica com a sua comunidade e é encarregado de criar um elo entre a ESF e a população (BRASIL, 2012). 
Os riscos da vida sexual ativa na adolescência: o relato de uma capacitação em uma Estratégia de Saúde da Família

Partindo desse pressuposto, a autonomia dos ACS, que vem do conhecimento científico específico para exercerem seu papel, é de suma importância para a eficiência dos objetivos da ESF, sendo deles o contato inicial com a população e a principal forma de repasse de informações concernentes aos cuidados em saúde para as famílias, buscando evitar que elas sejam acometidas pelos agravos à saúde, provenientes da carência de instrução dos métodos preventivos. Deste modo, este relato se fundamentou se numa questão norteadora para estudo: Os ACS têm conhecimento suficiente para intervirem e instruírem adolescentes acerca dos riscos da atividade sexual na adolescência?

Compreendendo a importância de um trabalho de educação sexual em adolescentes, o objetivo deste trabalho foi relatar uma capacitação feita por acadêmicos de medicina a Agentes Comunitários de Saúde acerca dos riscos da vida sexual na adolescência em uma Estratégia de Saúde da Família da cidade de Montes Claros, MG.

\section{MATERIAIS E MÉTODOS}

Trata-se de um estudo do tipo relato de experiência, de caráter qualitativo, de uma intervenção feita por acadêmicos do curso de medicina de uma universidade de Montes Claros, MG em uma Estratégia de Saúde da Família da mesma cidade, em dezembro de 2016. As atividades foram desenvolvidas por 4 acadêmicos e haviam 5 Agentes Comunitários de Saúde presentes.

As atividades ocorreram na Estratégia de Saúde da Família Vila Telma durante o mês de dezembro de 2016 e foram divididas em duas partes: Exposição de Conhecimentos dos ACS e Capacitação. Inicialmente, os ACS expuseram verbalmente seus conhecimentos sobre educação sexual expondo os métodos preventivos de gravidez e DST, rotineiramente transmitidos à comunidade. Estratégia similiar foi adotada por Fioramonte et al., (2013), onde as ações de enfermeiros da ESF também foram avaliadas, porém, por entrevistas semiestruturadas e apontaram insegurança e despreparo, indicando necessidade de capacitação aos profissionais. Os ACS também interagiram com os acadêmicos, relatando experiências que vivenciam na ESF, tais como visitas às famílias com adolescentes com casos de DST e gestação sem planejamento, resultado da iniciação precoce e desprotegida na vida sexual, gerando problemas financeiros e familiares.Em seguida, os acadêmicos realizaram uma capacitação com o intuito qualificar os agentes para suas ações de prevenção e promoção da saúde de adolescentes. Os acadêmicos atuaram expondo dados científicos acerca da gravidez e DST, suas formas de prevenção e consequências na adolescência, ressaltando também aos agentes sobre a importância da sua 
Os riscos da vida sexual ativa na adolescência: o relato de uma capacitação em uma Estratégia de Saúde da Família

atuação, haja vista a sua proximidade com a população e a relevância de suas orientações aos adolescentes vulneráveis e aos que, de fato, vivenciam as situações decorrentes do sexo desprotegido.

\section{RESULTADOS E ANÁLISES}

A partir das atividades desenvolvidas na capacitação, foi possível observar que os agentes de saúde não tinham autonomia para atuarem sobre o tema, ao revelarem não possuir conhecimento suficiente para instruir a população adscrita à ESF acerca dos riscos da vida sexual ativa na adolescência. Alguns agentes relataram que, em alguns casos, ao se depararem com adolescentes que estavam vivenciando alguma enfermidade decorrente da atividade sexual desprotegida, apenas agendavam consultas sem dar algum tipo de orientação, evidenciando a necessidade de intervenção a nível instrucional nos casos de jovens atingidos por alguma doença ou em situação de risco. A Estratégia de Saúde da Família busca reorganizar a atenção básica do país desde 1994, atuando com equipes multidisciplinares e buscando fortalecer a premissa de levar os cuidados em saúde à população adscrita, sendo canal de acesso aos serviços (MINISTÉRIO DA SAÚDE, 2011). Sendo assim, intervenções dos ACS ao defronte com casos provenientes da atividade sexual na adolescência mostram-se pertinentes, ao passo que, os estágios de uma doença podem se desenvolver durante a espera à uma consulta médica.

A capacitação dos acadêmicos contou com atividades educativas e possibilitou uma contribuição para a ampliação do conhecimento dos ACS sobre temas relevantes à problemática dos riscos associados à sexualidade na fase da adolescência, tais como: gestação e o contágio à DST. Foi exposto aos agentes a importância do perfil preventivo, onde se procura evitar o problema antes que ele se concretize sendo necessário, para que isso seja possível, ter uma postura ativa na divulgação de informações sobre o assunto aos grupos pré-dispostos.

Os agentes relataram que casos de gravidez não planejada e de doenças sexualmente transmissíveis são recorrentes na região, que se situa numa área periférica e com baixo poder aquisitivo. Alguns ACS afirmaram que adolescentes grávidas muitas vezes interrompem os estudos por causa da gestação e nem todas retornam à escola. Um estudo com escolares em Goiás averiguou uma associação entre condição socioeconômica mais baixa e a prática sexual (SASAKI et al., 2015), além de verificar que a prevalência de relação sexual alguma vez foi elevada entre os estudantes. Adolescentes considerados mais vulneráveis, caso sejam acometidos por alguma DST ou gravidez não planejada, podem sofrer agravos substanciais relacionados, principalmente, ao seu desenvolvimento, visto que essa é uma fase conhecida pelo amadurecimento físico, psicológico e social. Dada a frequência de casos na ESF avaliada, torna-se 
Os riscos da vida sexual ativa na adolescência: o relato de uma capacitação em uma Estratégia de Saúde da Família

pertinente uma atenção cuidadosa não apenas pela equipe de saúde da família, mas também pelos pais, que podem desenvolver um diálogo favorável ao acolhimento e a afirmação dos filhos nesta fase de construção da identidade sexual e definição do espaço social do homem e da mulher (DAVIM et al., 2009).

Um estudo evidenciou que fatores como consumo de álcool, tabaco, drogas ilícitas e envolvimento em situações de violência estão vinculados à idades de iniciação sexual precoces em adolescentes ( $\geq 15$ anos), revelando que a iniciação sexual pode estar associada à outras condutas (MADKOUR, 2010). Baumann (2011) apontou que as chances de acometimento de problemas de saúde na adolescência e nas subsequentes fases da vida foram maiores quanto menor foi a idade de inicio da vida sexual e o número de parceiros tendeu a aumentar quanto mais cedo foi a iniciação sexual. Deste modo, salienta-se que novas estratégias focadas na educação sexual, informação e prevenção, devem ser aplicadas desde o início da adolescência, por ser esta fase da vida determinante para a saúde da vida adulta.

Os ACS demonstraram conhecimento insuficiente sobre o assunto durante a atividade, mas questionaram a relevância de suas interferências na promoção da saúde. Sabe-se que o agente possui papel fundamental, não só no tratamento, mas também na prevenção realizada através do repasse de informações e esclarecimentos à população adscrita à Unidade Básica de Saúde (UBS). Ao final da capacitação, os ACS mostraram-se mais motivados por terem adquirido mais conhecimento e por poderem atuar com mais segurança e oferecerem maior cuidados com a população local.

Dentre as funções que o Ministério da Saúde (BRASIL, 2012) atribui aos ACS que, em suma, visam o acompanhamento e a integração entre a população credenciada e a UBS, destacam-se outras funções importantes como: realizar atividades programadas e de atenção à demanda espontânea; estar em contato permanente com as famílias, desenvolvendo ações educativas visando à promoção da saúde, à prevenção das doenças e ao acompanhamento das pessoas com problemas de saúde. A Estratégia de Saúde da Família é uma estratégia de expansão, qualificação e consolidação da atenção básica. Para tanto, os ACS devem estar preparados e munidos de conhecimento dos métodos de prevenção e promoção à saúde, para então ter autonomia e capacidade para exercer o seu papel em plenitude.

A realização deste trabalho permitiu observar que a ESF avaliada pode impactar ainda mais a população, disseminando e preparando os profissionais com maior acervo de informações com intuito de promover a saúde ao utilizar do maior elo entre a Unidade Básica de Saúde e a comunidade - o Agente Comunitário de Saúde. Quanto mais saber ele portar acerca dos métodos de promoção à saúde, que envolvem o conhecimento peculiar das doenças e formas preventivas, 
Os riscos da vida sexual ativa na adolescência: o relato de uma capacitação em uma Estratégia de Saúde da Família

maior será a sua contribuição para o alcance efetivo dos objetivos das Estratégias de Saúde da Família.

\section{CONSIDERAÇÕES FINAIS}

Considera-se que a intervenção gerou novos aprendizados e impactou positivamente a Estratégia de Saúde da Família estudada, munindo os agentes de saúde com informações técnicas, dentre elas, métodos de prevenção às DST e gravidez não planejada, além de dados científicos sobre os riscos a que os adolescentes estão suscetíveis ao se exporem ao sexo desprotegido.

Os agentes de saúde que compõem a equipe da Estratégia de Saúde da Família são considerados porta de entrada ao sistema de saúde público e são fundamentais na atenção básica por promoverem saúde e minimizarem possíveis agravos. Por terem uma maior proximidade com a comunidade local, são um elo importante e facilitam a acessibilidade dos usuários à rede. Portanto, os agentes podem atuar promovendo atividades de educação em saúde aos adolescentes em alcance, incentivando o uso de preservativos e de outros métodos anticoncepcionais e preventivos, além de instruir sobre as DST.

O papel do ACS não deve se restringir apenas ao direcionamento ao atendimento médico, mas também à instrução do indivíduo sobre as formas profiláticas às doenças, explicando sobre as possíveis enfermidades e as consequências das suas transmissões, além de métodos que previnam a gravidez, com a finalidade de evitar reincidências e minimizar problemas atuais e futuros de saúde local. Desta maneira os ACS cumprirão as diretrizes direcionadas pelo Ministério da Saúde e, se com sucesso executadas, farão parte de grandes mudanças no cenário atual da saúde do Brasil.

Considera-se também que estudos sobre fatores associados ao início da atividade sexual na adolescência podem ser de grande valia para a aplicação de políticas de saúde pública apropriadas e focadas na prevenção e instrução, buscando garantir a qualidade de vida e a saúde dos adolescentes.

\section{REFERÊNCIAS}

BAUMANN, P.; BÉLANGER, R. E.; AKRE, C.; SURIS, J. C. Increased risks of early sexual initiators: time makes a difference. Sex Health, v. 8, n. 3, p. 431-435, 2011.

BRASIL, M. da S. Política Nacional de Atenção Básica - PNAB. Ministério da Saúde, Legislação em Saúde, Brasília, Série E, 2012.

BRASIL. Recomendações para a Atenção Integral a Adolescentes e Jovens Vivendo com HIV/Aids. Ministério da Saúde, Brasília, DF, 1ª Edição, 2013. 
Os riscos da vida sexual ativa na adolescência: o relato de uma capacitação em uma Estratégia de Saúde da Família

CHAVES, A. C. P.; BEZERRA, E. O.; PEREIRA, M. L. D.; WAGNER, W. Conhecimentos e atitudes de adolescentes de uma escola pública sobre a transmissão sexual do HIV. Rev. Brasileira de Enfermagem, v. 67, n.1, p. 48-53, 2014.

CRUZEIRO, A. L. S.; SOUZA, L. D. M.; SILVA, R. A.; PINHEIRO, R. T.; ROCHA, C. L. A.; HORTA, B. L. Comportamento sexual de risco: fatores associados ao número de parceiros sexuais e ao uso de preservativo em adolescentes. Ciên. Saúde Colet.; v. 15, n. 1, p. 1149-1158, 2010.

DAVIM, R. M. B.; GERMANO, R. M.; MENEZES, R. M. V.; CARLOS, D. J. D. Adolescente/Adolescência: Revisão Teórica sobre uma fase crítica da vida. Rev. Rene. Fortaleza, v. 10, n. 2, p. 131-140, 2009.

DOS SANTOS, K. T.; SALIBA, N. A.; MOIMAZ, S. A. S.; ARCIERI, R. M.; CARVALHO, M. L. Agente comunitário de saúde: perfil adequado à realidade do Programa de Saúde da Família? Ciência \& Saúde Coletiva, v. 16, supl. 1, p. 1023-1028, 2011.

FIORAMONTE, A.; BRESSAN, B. F.; SILVA, E. M.; NASCIMENTO, G. L.; BURIOLA, A. A. Cuidado à pessoa com transtorno mental e sua família: atuação do enfermeiro na ESF. Ciência e Cuidado Saúde, v. 12, n. 2, p.315-22, 2013.

GONÇALVES, H.; KNAUTH, D. Aproveitar a vida, juventude e gravidez. Revista de Antropologia, São Paulo, v.8, n. 2, p. 625-643, 2006.

GRADIM, C. V. C.; FERREIRA, M. B. L.; MORAES, M. J. O perfil das grávidas adolescentes em uma unidade de saúde da família de Minas Gerais. Rev. APS, Juiz de Fora, v. 13, n.1, p. 5561, 2010.

IBGE, Instituto Brasileiro de Geografia e Estatística. Pesquisa Nacional de Saúde do escolar 2015. Instituto Brasileiro de Geografia e Estatística. Ministério da Saúde, Rio de Janeiro, 2016.

MADKOUR, A. S.; FARHAT, T.; HALPERN, C. T.; GODEAU, E.; GABHAINN, S. N. Early adolescent sexual initiation as a problem behavior: a comparative study of five nations. $\mathbf{J}$. $\begin{array}{lllllll}\text { Adolesc. Health, } & \text { v. 47, n. } 4, \quad \text { p. } & 389-398, & \end{array}$

6. Ministério da Saúde (BR). Portaria no 2.488, de 21 de outubro de 2011. Política Nacional de Atenção Básica. [online]. 2011 [acesso em: 2013 out 31]. Disponível em: http://189.28.128.100/dab/docs/publicacoes/geral/pnab.pdf

SÁNCHEZ, A. I. M.; BERTOLOZZI, M. R. Pode o conceito de vulnerabilidade apoiar a construção do conhecimento em Saúde Coletiva? Ciência \& Saúde Coletiva, Rio de Janeiro, v. 12, n. 2, p. 319-324, 2007.

SASAKI, R. S. A.; LELES, C. R.; MALTA, D. C.; SARDINHA, L. M. V.; FREIRE, M. C. M. Prevalência de relação sexual e fatores associados em adolescentes escolares de Goiânia, Goiás, Brasil. Ciênc. Saúde Coletiva [online], v. 20, n. 1, p. 95-104, 2015. 
Os riscos da vida sexual ativa na adolescência: o relato de uma capacitação em uma Estratégia de Saúde da Família

SAWYER, M.; AFIFI, R. A.; BEARINGER, L. H.; BLAKEMORE, S. J.; DICK, B.; EZEH, A. C.; PATTON, G. C.; Adolescence: a foundation for future health. The Lancet, v. 379, n. 9826, p. 1630-1640, 2012.

Recebido em: 10/05/2017

Aceito em: 29/06/2018 